\title{
THE SEASONAL INCIDENCE OF ONSET AND EXACERBATIONS IN MULTIPLE SCLEROSIS
}

\author{
BY
}

\section{KURT SCHAPIRA}

From the Department of Neurology, Royal Victoria Infirmary, Newcastle upon Tyne

That the disease is significantly more common in the northern part of the northern hemisphere is one of the few epidemiological observations about multiple sclerosis which can be regarded as reasonably well established. The cause for this increased incidence in colder climates remains entirely obscure: an increased prevalence of upper respiratory infections or of vasospastic disorders under such conditions are amongst the possible mechanisms which have been speculatively invoked, but there is no reliable evidence in either direction.

For these reasons it was considered of interest to study the case histories of a large number of patients with multiple sclerosis personally examined in the north-east of England, to ascertain whether there was any seasonal incidence, either in the onset or in exacerbations of the established disease. There is little previous information on these lines in the literature of the subject, and Limburg, in the volume on multiple sclerosis and the demyelinating diseases, published by the Association for Research in Nervous and Mental Disorders in 1950 , discusses the difficulty in assessing seasonal variations retrospectively on the basis of mortality and morbidity statistics, and suggests further observations drawn from clinical protocols, as in the present study. Hopkins and Swank (1955), in a study of patients in north-eastern Canada, found no monthly variation in incidence but noted a significant correlation with diurnal temperature range.

In many instances the onset of multiple sclerosis cannot be dated within a month, and in any case it is unlikely that the onset of symptoms coincides exactly with the initiation of the pathological lesion. It is also possible that new symptoms may be caused by one or two strategically situated lesions amongst many simultaneously initiated or re-activated, and that some pathological exacerbations may even be entirely asymptomatic. However, both the onset of symptoms and, particularly the identifiable acute exacerbations of the disease, presumably indicate at the least a critical activation of the disease processa pathological as well as a clinical exacerbation.

Quite often, the progress of the disease is insidious throughout. Such cases were excluded from the present series but an analysis of 700 case histories yielded relevant information in 514 instances, 246 in which the onset could be clearly dated, and 268

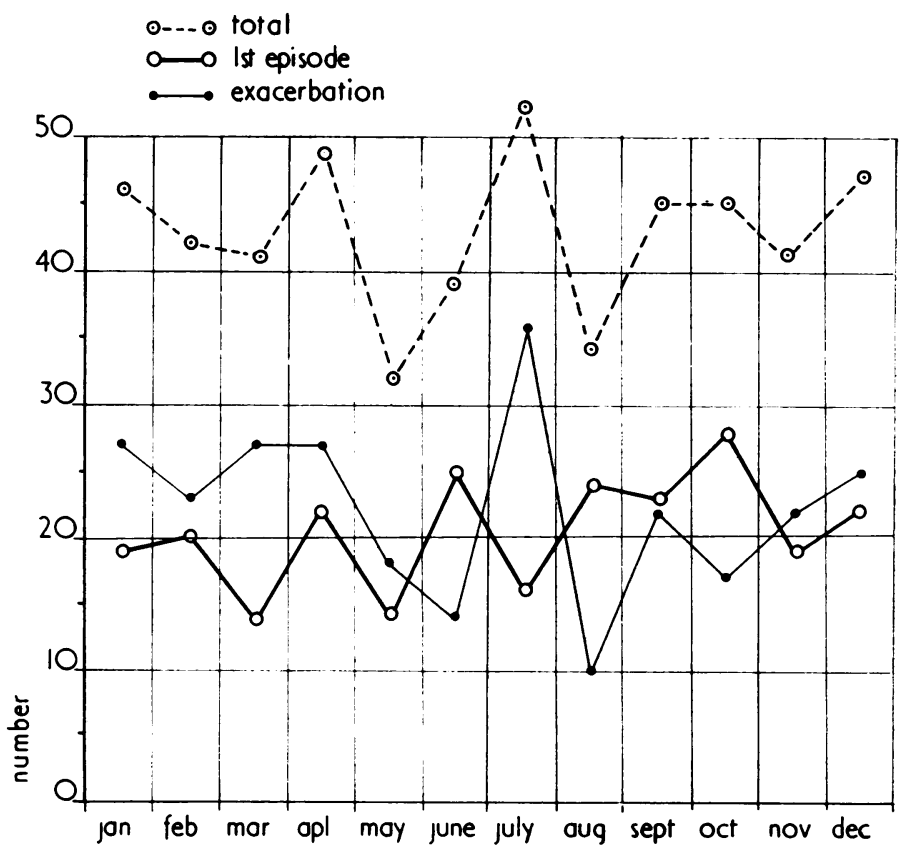

Incidence by MONTH of Multiple Sclerosis (analysis of 514 episodes) 
identifiable acute exacerbations. The monthly distribution of these incidences, separately and in combination, is indicated in Fig. 1.

There is little difference in the incidence per month of initial episodes ( $x^{2}$ with 11 degrees of freedom $=10.617$-not significant). More exacerbations, however, occurred in July than in any other month, but this increase in July is balanced by a decrease in June and August. When the incidence for threemonthly periods throughout the year is compared, there is no evidence of seasonal preponderance either for total episodes (January to March, 130;
April to June, 120; July to September, 131; October to December, 133) or for initial episodes or exacerbations.

The author wishes to thank the North-East Multiple Sclerosis Trust for the financial support which has made this work possible, and Dr. Henry Miller for suggesting the subject of the present investigation.

\section{REFERENCES}

Limburg. C. L. (1950). Res. Publ. Ass. nerv. ment. Dis.. XXVIII,

Hopkins, C. E., and Swank, R. L. (1955), A.M.A. Arch. Neurol. Psychiat., 74, 203.

\section{THE AUGUST (1959) ISSUE}

The August (1959) issue contains the following papers:-

Memories of Hugh Cairns Sir Geoffrey Jefferson.

Changes in Human Muscles after Permanent Tenotomy. S. Sunderland and J. O. Lavarack.

The Range of Conduction Velocity in Normal Motor Nerve Fibres to the Small Muscles of the Hand and Foot. P. K. Thomas, T. A. Sears, and R. W. Gilliatt.

A Clinical and Experimental Study of the Effects of Exercise on Motor Weakness in Neurological Disease. J. A. R. Lenman.

A Case of Spontaneous Dissecting Aneurysm of the Internal Carotid Artery. R. McD. Anderson and M. M. Schechter

Ruptured Posterior Fossa Aneurysms and their Surgical Treatment. Helen Dimsdale and Valentine Logue. The Association of Cerebral Angiomas with Intracranial Aneurysms. J. S. Boyd-Wilson.

Congenital Arteriovenous Fistula with Aneurysm of the Great Cerebral Vein and Hydrocephalus Treated Surgically. James B. Gibson, Alexander R. Taylor, and Alan E. Richardson.

Traumatic Thrombosis of the Internal Carotid Artery. T. D. R. Hockaday.

Clinical and Electroencephalographic Investigations in Myoclonic Cerebellar Dyssynergia. A. Kreindler, E. Crighel, and I. Poilici.

Is Lumbar Puncture Harmful in Multiple Sclerosis? Kurt Schapira.

Slowness in Schizophrenia. Arthur Harris and Maryse Metcalfe.

The Use of an Object Sorting Test in Elucidating the Hereditary Factor in Schizophrenia. N. McConaghy.

A Clinical and Biochemical Study of a Trial of Iproniazid in the Treatment of Depression. C. M. B. Pare and M. Sandler.

A Cerebrospinal Fluid Bank. Harold C. Voris and Peter J. Talso.

Book Reviews.

A number of copies are still available and may be obtained from the Publishing Manager, British Medical Association, Tavistock Square, W.C.1, price 17s. 6 . 